\title{
A COMUNIDADE DE PRÁTICA SOB A ÓTICA DA ANÁLISE DE REDES SOCIAIS: UMA APLICAÇÃO EM UMA COOPERATIVA BRASILEIRA
}

\author{
Andréia Maria Kremer $^{1}$, Wesley Batista Akahoshi ${ }^{1}$ \& Rafael Todescato Cavalheiro ${ }^{\text {* }}$
}

\section{RESUMO}

Kremer, A.M, AKAhOSHI, W.B, CAVAlHEIRO, R.T. A Comunidade De Prática Sob A Ótica Da Análise De Redes Sociais: Uma Aplicação Em Uma Cooperativa Brasileira. Perpectivas Online: Humanas \& Sociais Aplicadas, v.7, n.20, p.28-42, 2017.

As relações sociais e cooperação ganharam destaque como mecanismo propulsor da transmissão de conhecimentos nas redes. Nesse contexto, esse estudo relaciona os conceitos de Comunidade de Prática (CoP) e de análise de redes sociais com o objetivo de identificar, caracterizar e analisar a rede social das CoPs na rede formada pelos membros de uma cooperativa brasileira. Os dados foram coletados através de questionários com questões fechadas e abertas e analisados por meio da metodologia de análise de redes sociais. Os resultados revelaram que no interior da rede social formada pelos membros da cooperativa, um grupo possuía destaque com maior coesão e maior centralidade, caracterizado como uma CoP. Esse grupo apresentou características de uma CoP tanto antes da formação da cooperativa como também após sua formação. Os resultados são confirmados pelos indicadores de análise de redes sociais.

Palavras-chave: Redes sociais; Comunidade de Prática; Cooperativa.

\begin{abstract}
Social relations and cooperation have gained prominence as a driving force for the transmission of knowledge in networks. In this context, this study relates the concepts of Community of Practice $(\mathrm{CoP})$ and analysis of social networks with the objective of identifying, characterizing and analyzing the social network of CoPs in the network formed by members of a Brazilian cooperative. The data were collected through questionnaires with closed and open questions and

analyzed through the methodology of social network analysis. The results revealed that within the social network formed by the members of the cooperative, a group had prominence with greater cohesion and greater centrality, characterized as a CoP. This group presented characteristics of a $\mathrm{CoP}$ both before the formation of the cooperative and also after its formation. The results are confirmed by the indicators of social network analysis.
\end{abstract}

Keywords: Social networks; Community of Practice; Cooperative.

\footnotetext{
${ }^{1}$ Universidade Federal da Grande Dourados - Faculdade de Administração, Ciências Contábeis e Economia (FACE), Campus Universitário, Dourados - MS, CEP:79804-970, Brasil.

(*)e-mail: rafaeltodescato@hotmail.com

Data de chegada: 20/05/2017 Aceito para publicação: 19/09/2017
} 


\section{INTRODUÇÃO}

As estruturas organizacionais têm apresentado mudanças no decorrer dos anos, na economia como um todo, evidenciados principalmente nas estruturas analíticas da cadeia produtiva. No agronegócio, esse dinamismo nas estruturas e nos mercados, tem tornado necessária a busca por alternativas de sobrevivência que possibilitem competitividade as organizações e aos produtores rurais, um dos exemplos é a inserção em novas cadeias produtivas.

Essas mudanças acabam forçando as organizações e os produtores rurais a estabelecer parcerias baseadas na cooperação com outras organizações ou produtores. Principalmente no meio rural, em que os produtores possuem maiores dificuldades no acesso a conhecimentos técnicos e práticos, causado pela limitação de recursos, apoio, e também pela questão cultural e educacional dos produtores rurais. Essas parcerias surgem como meio de acesso a informações, troca de experiências e troca de práticas necessárias para a manutenção de suas propriedades.

Para grupos como esses, Schneider $(2004$, p. 2) mostra que uma solução pode ser encontrada por meio do cooperativismo, afirmando que "as cooperativas são uma resposta para os problemas impostos pela globalização". O termo cooperativa vem de cooperação, e essa cooperação sempre existiu nas sociedades humanas visando, sobretudo, a sobrevivência. Comunidades se uniam a milhares de anos, com o intuito de atingir objetivos e propósitos que sem cooperação seria muito difícil realizarem, ou seja, a cooperação é um processo social com raízes milenares, utilizada desde o início buscando o fortalecimento de um grupo.

Ainda nesse contexto, a cooperação é, segundo Gianezini (2010, p. 06), “[...] uma relação de colaboração, auxílio, trabalho mútuo e de trocas recíprocas entre homens. É um atributo das relações sociais que precisa ser valorizado tanto quanto é importante". Deste conceito apresentado por Gianezini, verifica-se a importância das relações sociais como precursor dessa troca de conhecimentos e experiências. Diante dessa importância, este estudo relaciona três conceitos com bases muito fortes na cooperação e nas relações sociais: cooperativa, redes sociais e comunidades de prática $(\mathrm{CoP})$.

A relação entre os três conceitos ocorre porque todos têm como base de sustentação, as relações sociais, com o individuo e suas interações. A cooperativa, como representação de uma alternativa para união de vários agentes em busca de objetivos comuns, com um espaço que proporciona a troca de experiências, saberes e informações, acaba por configurar-se como uma rede social.

A cooperativa também pode ser um espaço para surgimento de comunidades de prática, que na visão de Souza-Silva e Schommer (2008, p. 109) "é um grupo de pessoas que se aglutinam entre si para se desenvolverem em um domínio do conhecimento, vinculado a uma prática específica”. As CoP podem surgir no interior de uma cooperativa, ou podem ser a base inicial para uma cooperativa, pela organização e formalização de um grupo que se juntaram para troca de conhecimentos. Sendo assim, fica claro no conceito de CoP, a importância das relações sociais como sustentador da cooperação e compartilhamento de conhecimentos no grupo.

Pretende-se nesse estudo responder as seguintes questões: as relações sociais da CoP podem ser analisadas pela metodologia de análise de redes sociais? Em quais aspectos a análise de redes sociais pode fortalecer a integração ou a melhoria do aprendizado na CoP? E como as características da CoP podem ajudar na consolidação da cooperativa? Com vistas a abordar conjuntamente os três conceitos e a responder as questões abordadas anteriormente, objetiva-se, no presente estudo, identificar, caracterizar e analisar a rede social das CoP's, na rede formada pelos membros de uma cooperativa brasileira.

A estruturação do estudo seguirá da seguinte forma: além dessa parte introdutória, será realizada uma fundamentação teórica, abordando as redes de cooperação, a aprendizagem e sua relação com as redes de cooperação e a comunidade de prática. Logo após seguirá a metodologia utilizada, as análises e resultados do estudo, as considerações finais, finalizando com as referências utilizadas como base para a realização do estudo.

Persp. online: hum. \& sociais aplicada., Campos dos Goytacazes, 20 (7), 28-42, 2017

seer.perspectivasonline.com.br 


\subsection{Redes de cooperação}

Os estudos sobre a organização empresarial e suas formas de obtenção de vantagens competitivas por meio da cooperação entre empresas evoluem constantemente. No agronegócio, as estruturas organizacionais evoluíram, iniciando com o conceito de agribusiness, proposto por Davis e Goldberg (1957), que afirmavam que não era mais possível analisar a economia nos moldes tradicionais, com setores isolados que fabricavam insumos, processavam os produtos e os comercializavam. Os autores foram os precursores do agribusiness com visão sistêmica, que vai desde o produtor rural até o consumidor final.

A evolução conceitual com base no conceito de agribusiness passou por diversas correntes teóricas, que defenderam diferentes estruturas de análise do agronegócio no decorrer do tempo, evoluindo até chegar à abordagem de redes, motivada pela necessidade das empresas atuarem de forma conjunta e associada. $\mathrm{O}$ conceito de redes, advindo principalmente da sociologia, apresenta uma corrente teórica que prioriza o desenvolvimento empresarial por meio da associação, do compartilhamento, da troca e da ajuda mútua. Em concordância com essas características das redes, Fensterseifer $(2000$, p. 5) conceitua a rede como um "complexo de relações cooperativas que dinamizam a ação de seus agentes em torno de objetivos comuns ou complementares".

Para as ciências sociais as redes designam normalmente movimentos pouco institucionalizados que reúnem grupos ou indivíduos em associações com as mais distintas características. As redes, nesse contexto, são “(...) compostas de indivíduos, grupos ou organizações, e sua dinâmica está voltada para a perpetuação, a consolidação e o desenvolvimento das atividades de seus membros" (MARTELETO, 2001, p. 73). Segundo esse mesmo autor, as redes são organismos informais onde um conjunto de indivíduos autônomos unem recursos e ideias em prol de interesses comuns.

Para Silva (2010, p. 21) "as redes sociais são estruturas polifórmicas formadas necessariamente por atores sociais ligados por laços formais ou informais". Os conceitos de redes, em sua maioria, consideram como propulsor da organização em rede, a necessidade de indivíduos se associarem a outros com objetivos comuns, e para tanto se utilizam das relações sociais como mecanismo para escolha dos componentes da rede e para manutenção dos objetivos comuns.

De acordo com Fontes e Eichner (2004), as redes sociais são oriundas das interações sociais estabelecidas pelos indivíduos. Os autores afirma que esses processos complexos são primordiais para o entendimento dos fenômenos subjacentes à organização da sociedade. A possibilidade de analisar a posição dos indivíduos em suas relações permite identificar as suas possibilidades de acessar os recursos e identificar o papel que desempenha na sociedade como um todo (FONTES; EICHNER, 2004).

As primeiras abordagens das relações sociais que ligam os atores em forma de redes podem ser atribuídas inicialmente á corrente teórica da Nova Sociologia Econômica, que iniciou-se na década de 1970, com a tese de doutorado de Mark Granovetter, intitulada "Getting a Job" (1974). Granovetter adotou o conceito de embeddedness, que afirma que as relações sociais imersas possuem três características que controlam as expectativas e o comportamento dos agentes nas relações: a confiança, a troca de informações e a resolução conjunta de problemas (UZZI, 1997).

Para Uzzi (1997), as relações sociais entre os atores, organizações e instituições se desenvolvem a partir de uma rede de referências ou de relações anteriores, formando uma rede de confiança nas relações e a troca de informações ao longo do tempo fortalece os vínculos de troca e de resolução de problemas em conjunto. Sendo assim, as relações organizacionais podem afetar positivamente os resultados organizacionais e econômicos. Além disso, as relações cooperativas ainda podem funcionar como uma ferramenta de controle social, influenciando a magnitude do conhecimento trocado e a eficiência na qual o conhecimento é compartilhado em relações de cooperação (PARKHE, 1998).

Diante da afirmação de Parkhe (1998) e de Uzzi (1997), verifica-se que as redes de relações pessoais, principalmente as baseadas em confiança entre os agentes, possuem um alto potencial de aprendizagem organizacional para os individuos e organizações inseridas na rede de cooperação. 


\subsection{Aprendizagem organizacional e as redes de cooperação}

O termo aprendizagem, pela sua natureza cognitiva e multidisciplinar, não possui uma definição única adotada, assumindo definições distintas em diferentes áreas do conhecimento, independente da abordagem individual ou organizacional. Segundo Tsang (1997), apesar da crescente utilização do termo aprendizagem organizacional, ainda existem poucas ideias consensuais sobre definições, aplicações, contextualizações e metodologias.

Dyer e Nobeoka (2000) caracterizam as rotinas de apren $\neg$ dizagem como um padrão regular de interações entre os indivíduos que permitem a transferência, recombinação ou criação de conhecimento especializado. Diante deste conceito, verifica-se que a integração em rede surge como propulsor da aprendizagem organizacional, visto que a abertura e a riqueza das redes possibilitam a criação e o compartilhamento de conhecimento. A organização não pode criar conhecimentos por si só, pois necessita da interação do indivíduo e entre os indivíduos pertencentes à organização (NONAKA; TAKEUCHI, 1997).

Dyer e Nobeoka (2000) afirmam que a capacidade de aprendizagem de uma empresa vai além da sua capacida $\neg$ de como entidade única, isolada, necessitando para tanto da análise da rede na qual a empresa está inserida. Para os autores, a empresa deve ser capaz de criar e se posicionar nestas redes de aprendizagem criando rotinas para o compartilhamento de conhecimento entre os membros participantes. Com base nos conceitos apresentados por Dyer e Nobeoka e por Nonaka e Takeuchi, de que a aprendizagem ocorre de forma coletiva, com a interação entre os indivíduos, pode-se associar o conceito de aprendizagem interorganizacional, que, segundo Larsson et al. (1998, p. 287), "[...] pode, então, ser vista como uma aquisição coletiva de conhecimento entre um conjunto de organizações".

Larsson et al. (1998) afirmam que a aprendizagem interorganizacional acontece por meio da transferência do conhecimento existente de uma organização para outra, como também criando conhecimento completamente novo através da interação entre as organizações. Porém, para que o processo de aprendizagem interorganizacional seja efetivo e não sofra interferências oportunistas de algum dos envolvidos, é necessário que este, se alicerce em relações de confiança, transparência e cooperação, visando a aprendizagem do grupo como um todo, e não somente em nível individual. Nesta lógica, segundo Larsson et al. (1998), a transferência e a criação de conhecimento requerem níveis de transparência e receptividade entre as organizações que estabelecem relações de parceria.

Além dos aspectos abordados por Larsson et al. (1998), algumas variáveis também podem influenciar na forma e na intensidade da aprendizagem interorganizacional. De acordo com Levinson e Asahi (1995) os fatores culturais, estruturais e tecnológicos influenciam na aprendizagem oganizacional. A cultura influencia o processo de aprendizagem ao relacioná-la com a abertura da organização para mudanças, visto que organizações mais flexíveis possuem maior sucesso no processo de aprendizagem. A tecnologia, por sua vez, facilita tanto o processo de aprendizagem através da transferência de conhecimento como a conexão mais prática com as demais organizações.

Com relação à estrutura, foco deste estudo, Levinson e Asahi (1995) afirmam que essa variável pode ser analisada através dos itens: número de organizações da rede, a posição de uma organização participante na estrutura, o nível de conexões entre as organizações e o embeddedness da estrutura na rede. Esses fatores são importantes na análise do processo de aprendizagem, visto que podem influenciar o processo de criação e transferência de conhecimentos.

Ao considerar a afirmação de Larsson et al. (1998) de que a aprendizagem interorganizacional acontece por meio da transferência do conhecimento existente através da interação entre as organizações ou indivíduos, é possível afirmar que essa rede de interação entre indivíduos pode caracterizar a formação de um grupo com o objetivo comum de socialização de conhecimento por meio de uma comunidade de prática.

Persp. online: hum. \& sociais aplicada., Campos dos Goytacazes, 20 (7), 28-42, 2017

seer.perspectivasonline.com.br 


\subsection{Comunidades de Prática}

As CoPs surgem em um contexto onde a metodologia de aprendizagem formal e sistemática não consegue atender necessidades de conhecimento relacionadas a fatos difíceis de serem relatados formalmente. Conforme afirmam Gropp e Tavares (2006, p. 22) "a prática, o fazer conjunto, cria mais do que produtos - cria conhecimento, criatividade, aprendizado - cria comunidades de prática”.

Dessa forma, podem-se definir as CoP como grupos de pessoas que compartilham uma preocupação, um conjunto de problemas, ou uma paixão sobre um tópico e que aprofundam seu conhecimento e experiência nesta área, interagindo continuamente (WENGER, et al. 2002). Gropp e Tavares (2006, p. 27) reforçam esse conceito ao afirmarem que as CoPs são estruturas auto organizadas com o objetivo de gerar conhecimento prático e que "é nelas que as interpretações e os significados são assimilados por seus constituintes, transformando-se no conhecimento estratégico tão procurado e possibilitando a criatividade e a inovação".

Não se pode confundir CoP com outras estruturas sociais, como grupo de amigos, departamentos formais, comunidades de interesse, entre outros. No entanto, é saudável e ideal o estreitamento entre as relações pessoais por meio de relacionamentos de amizade, já que estes podem ser capazes de promover a confiança e, assim, maior facilidade no compartilhamento de conhecimento.

Uma CoP, na visão de Souza-Silva e Schommer (2008, p. 109), "é um grupo de pessoas que se aglutinam entre si para se desenvolverem em um domínio do conhecimento, vinculado a uma prática específica". Nessa visão, fica claro que a CoP se dá inicialmente pela busca de um objetivo comum de um grupo de pessoas, com o intuito de desenvolverem conhecimento de uma prática, também comum ao grupo, e não por uma interação de amizade pré-existente.

Esse vínculo social pode ser adquirido com a convivência, fruto da CoP. Dessa forma, para se entender a noção de $\mathrm{CoP}$, três elementos básicos são necessários:

- O domínio: envolve compartilhamento de práticas que detêm um foco comum. O domínio cria uma base comum e um sentido de identidade comum. O domínio inspira membros a contribuir e a participar, orienta a sua aprendizagem, e dá sentido às suas ações. Conhecer os limites e as principais fronteiras do domínio permite que os membros decidam exatamente o que é compartilhamento de valor, como apresentar suas ideias, e quais as atividades a prosseguir permitindo também que reconheçam o potencial de ideias experimentais ou semiprontas (WENGER, et al., 2002).

- A comunidade: desenvolve relacionamentos para compartilhar conhecimentos. A comunidade cria o tecido social da aprendizagem. Uma comunidade forte promove interações e relações baseadas no respeito mútuo e confiança. A Comunidade é um elemento importante porque a aprendizagem é uma questão de pertencer, bem como, um processo intelectual envolvendo "coração e cabeça" (WENGER, et al., 2002).

- A prática é um conjunto de estruturas, ideias, ferramentas, informações, estilos, linguagem, histórias e documentos que são compartilhados entre os membros da comunidade. Esse corpo de conhecimentos e recursos compartilhados permite que a comunidade prossiga de forma eficiente para lidar com seu domínio (WENGER, et al., 2002).

As comunidades de prática têm o objetivo de desenvolver as capacidades de construção e troca de conhecimentos, de membros auto selecionados, que se fundem por meio da paixão, compromisso e identidade com o tema ou especialidade do grupo, podendo durar enquanto houver interesse dos membros do grupo. Para que obtenha o aprendizado através da prática é necessário "tornar-se membro de uma CoP, não implicando, simplesmente, uma questão de adquirir informações; requer, sim, disposição, conduta e perspectiva profissional" (BINOTTO; et al., 2007, p. 19). Sendo assim, para o melhor aproveitamento do conhecimento "oferecido" pela CoP é necessário estar inserido nela, recebendo e transmitindo conhecimento, construindo o conhecimento juntos, ou seja, através do coletivo.

Persp. online: hum. \& sociais aplicada., Campos dos Goytacazes, 20 (7), 28-42, 2017

seer.perspectivasonline.com.br 
Boyett e Boyett (1999) consideram que o desenvolvimento da CoP acontece por meio das redes informais, de conversas de corredor, de compartilhamento de informações, de formas de desempenhar o trabalho, da cooperação, entre outros. É uma comunidade dinâmica, versátil, e torna-se repositório de conhecimento.

A CoP é o compartilhar de ideias e experiências, é o vivenciar, é a cooperação em favor da criação e construção de conhecimento em comum. A CoP é, portanto, em sua essência, uma rede de cooperação informal, criada pela busca de objetivos comuns, de forma autônoma e regulada pelas relações de amizade e confiança entre os agentes. Sendo assim, uma análise da comunidade de prática através da metodologia de análise de redes sociais, pode fornecer informações importantes para a caracterização das CoPs.

\section{METODOLOGIA}

A pesquisa caracterizou-se por um estudo descritivo com abordagem quantitativa. Com a intenção de analisar uma CoP, sob uma perspectiva de análise de redes sociais informais de aprendizagem, optou-se por analisar as redes formadas por produtores rurais membros da Cooperativa de Piscicultores de Mundo Novo COOPISC, com sede no município de Mundo Novo, no estado de Mato Grosso do Sul.

Definido o escopo da pesquisa, foi realizada uma análise da estrutura da COOPISC, visando identificar os produtores que seriam abordados no estudo. A cooperativa possui 21 piscicultores cooperados, e para o estudo foi possível aplicar o questionário com uma amostra de $62 \%$ dos cooperados, ou seja, 13 piscicultores, devido a indisponibilidade de alguns piscicultores.

A coleta de dados foi realizada por meio da aplicação de questionários, com questões abertas e fechadas, com o objetivo de identificar os indivíduos e suas relações e, principalmente identificar se no interior da rede em análise existia uma CoP. Simultaneamente, foi realizada uma pesquisa documental com a finalidade de identificar o histórico e as características da cooperativa.

Concluída a coleta de dados, estes foram analisados por meio de um software de análise de redes sociais, ORA 2.3.6, calculando medidas de centralidade e prestígio de cada indivíduo na rede, identificando a posição de cada um nas relações. Com a finalidade de garantir o sigilo das informações e identidade dos pesquisados, foram atribuídos nomes fictícios aos cooperados para a análise dos dados. Por fim, representouse graficamente a estrutura da CoP com os recursos do software de análise de redes sociais.

\section{RESULTADOS E DISCUSSÕES}

\subsection{Perfil da COOPISC e dos cooperados}

O estudo foi realizado no município de Mundo Novo, localizado ao sul de Mato Grosso do Sul. O município possui aproximadamente 17 mil habitantes, e faz divisa com a cidade de Guaíra, estado do Paraná, e com a cidade paraguaia de Salto Del Guayrá. O município possui como característica principal o solo argiloso e a riqueza de águas que regam a região, propiciando condições bastante favoráveis ao desenvolvimento da atividade de piscicultura. A região possui um grupo forte de piscicultores, que desempenham posição de destaque no estado.

O grupo de piscicultores do município de Mundo Novo é organizado em forma de cooperativa, a COOPISC. A instituição foi constituída em dezembro de 1999 contando com 27 pequenos produtores do município com o objetivo principal de comercializar o pescado produzido pelos seus cooperados e de adquirir insumos para criação de peixes. Atualmente a cooperativa possui 21 membros.

No ano de 2000 a COOPISC recebeu a doação de um terreno da prefeitura municipal para a construção de um entreposto para beneficiamento do pescado. Em 2002 obteve o recurso para construção do entreposto, junto ao governo federal e através de financiamentos realizados pelo Banco do Povo. Porém, com o inicio do funcionamento do entreposto os cooperados perceberam algumas deficiências de estrutura e de equipamentos, concluindo que o local não possuía capacidade para processamento de toda a demanda. $\mathrm{O}$ entreposto entrou em funcionamento em março de 2008 e operou até abril de 2009, quando encerrou suas

Persp. online: hum. \& sociais aplicada., Campos dos Goytacazes, 20 (7), 28-42, 2017 seer.perspectivasonline.com.br 
atividades para estudos e reestruturação. perfil.

A análise inicial a ser feita é a caracterização dos produtores que compõe a COOPISC, quanto ao seu

Tabela 1: Perfil dos respondentes

\begin{tabular}{|c|c|c|}
\hline & Frequência & Frequência relativa \\
\hline \multicolumn{3}{|l|}{ Gênero } \\
\hline Masculino & 12 & $92,3 \%$ \\
\hline Feminino & 1 & $7,7 \%$ \\
\hline Total & 13 & $100,0 \%$ \\
\hline \multicolumn{3}{|l|}{ Faixa etária } \\
\hline Até 30 anos & 1 & $7,7 \%$ \\
\hline Entre 31 e 40 anos & 4 & $30,8 \%$ \\
\hline Entre 41 e 50 anos & 5 & $38,5 \%$ \\
\hline Mais de 50 anos & 3 & $23,0 \%$ \\
\hline Total & 13 & $100,0 \%$ \\
\hline \multicolumn{3}{|l|}{ Formação } \\
\hline Ensino fundamental incompleto ( $\left.1^{\circ} \mathrm{grau}\right)$ & 7 & $53,8 \%$ \\
\hline Ensino médio completo ( $2^{\circ}$ grau $)$ & 3 & $23,1 \%$ \\
\hline Ensino superior completo (graduação) & 3 & $23,1 \%$ \\
\hline Total & 13 & $100,0 \%$ \\
\hline
\end{tabular}

A Tabela 1 mostra que o nível de escolaridade entre os cooperados participantes é baixo, possuindo mais de $53 \%$ dos produtores apenas o ensino fundamental incompleto. Essa estatística fortalece a ideia que devido a baixa escolaridade, a dificuldade de criação de conhecimento de forma sistemática e explícita por meio de métodos tradicionais se torna, até certo ponto, pouco eficiente. Dessa forma, a criação de conhecimento através da CoP, torna-se uma alternativa para suprir essa deficiência.

Corroborando com a análise, no que tange a criação do conhecimento, pode-se relacionar o nível de escolaridade com a fonte de aprendizado referente à piscicultura e gestão da propriedade. Quando perguntado aos cooperados quais eram essas principais fontes de informação, com a possibilidade de indicação de mais de uma fonte, o resultado obtido se deu conforme a Tabela 2.

Tabela 2: Fonte de Aprendizado sobre Piscicultura e Gestão da Propriedade

\begin{tabular}{lcc}
\hline \multicolumn{1}{c}{ Fontes } & Frequência & Frequência relativa \\
\hline Jornais, revistas, livros & 2 & $6,7 \%$ \\
Internet & 2 & $6,7 \%$ \\
Assessoria técnica & 4 & $13,3 \%$ \\
Informações da Cooperativa & 2 & $6,7 \%$ \\
Produtores (troca de experiência) & 12 & $40,0 \%$ \\
Eventos, encontros e palestras. & 5 & $16,7 \%$ \\
Outros. & 3 & $10,0 \%$ \\
\hline
\end{tabular}

Considerando a Tabela 2, constata-se que a principal fonte de aprendizado é a troca de experiências entre os produtores, confirmando assim a ideia de Binotto $(2005$, p. 71$)$ que afirmou que "os pequenos agricultores preferem buscar informações de fontes que compartilham com seus valores e que lhes trarão noções de alinhamento com a sua atividade". Ainda se for considerado a assessoria técnica (13,3\%), informações da cooperativa (6,7\%), e os eventos, encontros e palestras (16,7\%) obtêm-se um total de $76,7 \%$ de fontes de aprendizado que independem da escolaridade, e que em sua maioria são transmitidos através do

Persp. online: hum. \& sociais aplicada., Campos dos Goytacazes, 20 (7), 28-42, 2017

seer.perspectivasonline.com.br 
compartilhamento das informações.

Já as opções de fontes como jornais, revistas e livros $(6,7 \%)$ e internet $(6,7 \%)$, depedem de um grau maior de escolaridade, haja vista a necessidade de leituras e interpretações, muitas vezes de difícil desenvolvimento para pessoas com um nível escolar baixo.

A experiência na criação de peixes tem, em média, entre os cooperados respondentes, cerca de 13 anos, o que coincide com o tempo de existência da COOPISC. No entanto, os valores absolutos variaram entre 5 e 21 anos de experiência na atividade. Sendo assim, existem produtores com diferentes níveis de experiência, considerando apenas os anos de atuação na atividade, e que antes da constituição da cooperativa já trabalhavam com a piscicultura.

Quanto ao tempo como associados à COOPISC, a média foi de aproximadamente 10 anos. No entanto, $67 \%$ dos respondentes eram pioneiros, ou seja, são membros desde a criação da cooperativa. Em seguida foram realizados questionamentos com a finalidade de identificar a relação da criação da cooperativa e a CoP. De acordo com a definição dada por Wenger et al. (2002), as CoP's são grupos de pessoas que compartilham uma preocupação, um conjunto de problemas, ou uma paixão sobre um tópico e que aprofundam seu conhecimento e experiência nesta área, interagindo continuamente. Nesse sentido, foi questionado aos respondentes se haviam reuniões informais, com objetivo de trocar experiências antes da criação da cooperativa e o resultado obtido foi que $50 \%$ dos participantes afirmaram que realizavam reuniões e $50 \%$ disseram que não realizavam.

Os produtores que realizavam reuniões afirmaram se reunir para tratar de assuntos relacionados à piscicultura, principalmente sobre formas de comercialização do pescado, buscando formas de canalizar recursos e de envolverem os produtores. Na opinião deles, através dessas reuniões surgiu a ideia da cooperativa como solução do problema de comercialização, ou seja, as reuniões contribuíram para a formação da COOPISC. Entre aqueles produtores que já se reuniam antes da formação da cooperativa, verifica-se que existe uma forte relação de troca de experiências, perceptível ao se questionar com quem mais aprendem, caracterizando, portanto, uma $\mathrm{CoP}$.

\subsection{Análise da CoP como rede social}

Os resultados anteriores confirmam a proposição de que as comunidades de prática são redes sociais, constituídas com base em cooperação e confiança entre os agentes. Com a finalidade de identificar a rede de troca de aprendizagem na COOPISC e consequentemente verificar se esta se caracteriza como uma CoP, foi solicitado aos participantes que indicassem o nome dos associados que trocam mais informações e que geram maior aprendizagem entre o grupo, em ordem de importância.

Dos questionários aplicados obteve-se o número mínimo de 4 indicações e máximo de 6 indicações. Com base nas respostas foi elaborada uma matriz quadrada de números, em que as linhas geram as relações de origem e as colunas as relações de destino. A rede de relações de troca de conhecimentos da COOPISC é composta por 14 indivíduos, sendo que um dos indivíduos do estudo não respondeu o questionário, mas foi citado por outros e, portanto, está incluso na rede por ser fonte de aprendizado de outros cooperados. A rede de relações de aprendizagem do COOPISC é apresentada na Figura 1. 


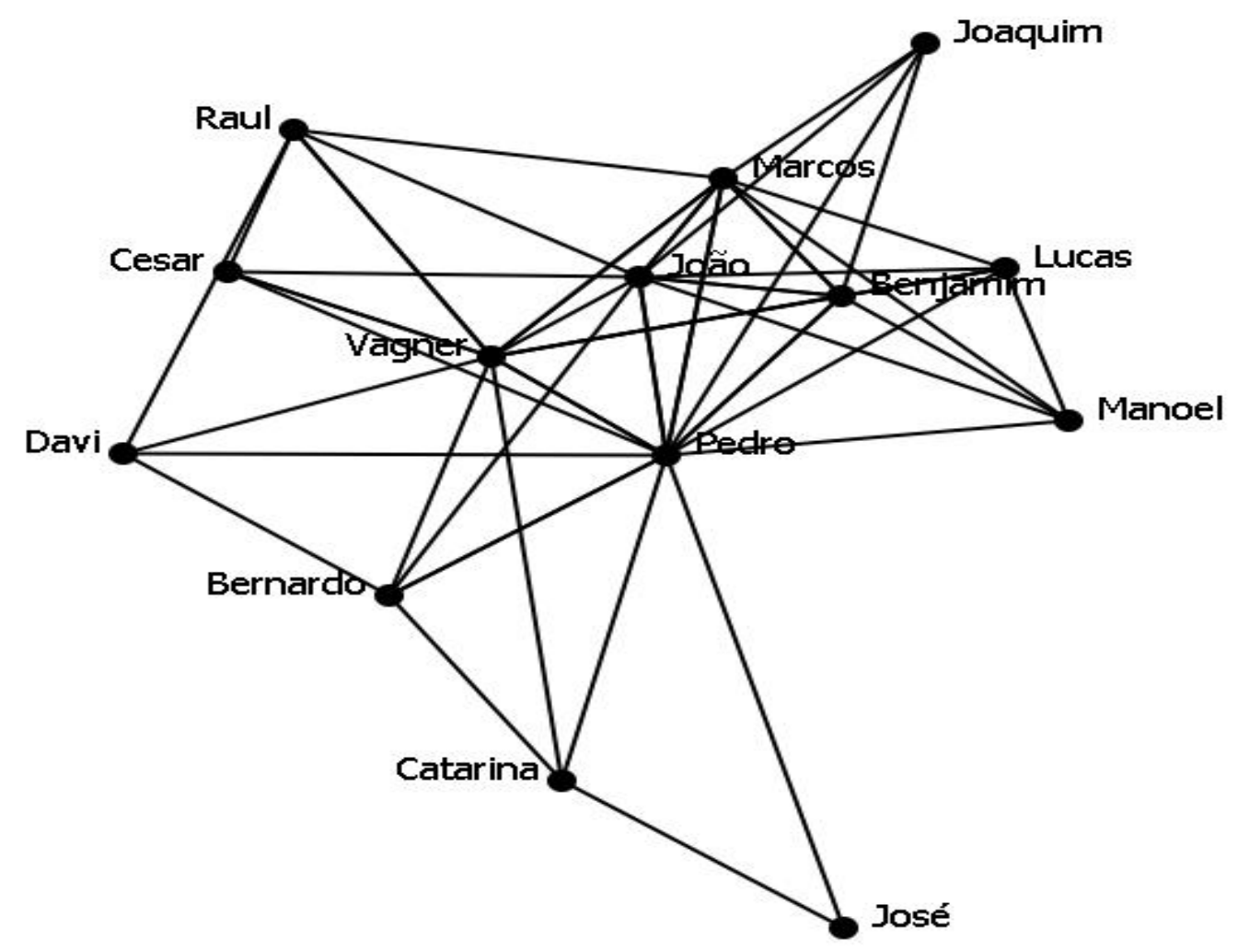

Figura 1: Rede de relações de aprendizagem da COOPISC

Seguindo o pressuposto teórico de Levinson e Asahi (1995), com relação à estrutura, a rede será analisada de acordo com as variáveis: número de organizações da rede, a posição de uma organização participante na estrutura, o nível de conexões entre as organizações e o embeddedness da estrutura na rede. Esses fatores são importantes na análise do processo de aprendizagem, visto que podem influenciar o processo de criação e transferência de conhecimentos. Em redes com menor quantidade de organizações, há menos habilidades para criação e identificação de novas ideias, porém, devido à proximidade dos agentes proporciona com maior facilidade o compartilhamento de informações. Além disso, os atores com maior centralidade em uma rede podem obter maior aprendizado, devido ao seu acesso maior as informações e maior confiança aos demais agentes (LEVINSON; ASAHI, 1995). A análise baseiase nos impactos dessas variáveis na geração e compartilhamento de informações e conhecimento, ou seja, uma análise de como a rede pode proporcionar a transmissão do conhecimento através das relações dos indivíduos.

A rede de relações sociais da COOPISC é uma rede curta, com poucos indivíduos envolvidos, sendo que somente foi considerado, nesse estudo, o elo de produtores da cadeia produtiva. Porém, apesar do número limitado de agentes, é possível analisar a rede pelas métricas adotadas por Levinson e Asahi (1995). A Tabela 3 apresenta as principais medidas de análise de redes da COOPISC.

Tabela 3: Medidas de análise de redes COOPISC

\begin{tabular}{lc}
\hline Medida & Valor \\
\hline Contagem de indivíduos & 14 \\
Numero de ligações & 56 \\
Densidade & 0,308 \\
Reciprocidade & 0,302 \\
Coeficiente de agrupamento & 0,551 \\
Centralidade de Intermediação & 0,138 \\
Centralidade de proximidade & 0,027 \\
\hline
\end{tabular}


O tamanho de uma rede consiste no total de relações efetivas ou potenciais em um grupo de indivíduos. Conforme mencionado anteriormente, a rede de relações de troca de conhecimentos da COOPISC é composta por 14 indivíduos. O tamanho da rede influencia o fluxo de conhecimentos ao considerar que em redes menores existe uma proximidade maior entre os agentes e, portanto, uma tendência à confiança entre eles. Conforme proposto por Levinson e Asahi (1995) redes mais dispersas ou maiores tendem a ser menos eficientes na transmissão da aprendizagem. O número de ligações também reflete o perfil da rede, visto que do total de ligações possíveis, estão sendo efetivadas 56 ligações, com uma média de 4 ligações por individuo.

A densidade de uma rede é obtida através da divisão do número de laços existentes pelo número total de possíveis laços. Para Granovetter (1973) existe uma relação entre a força dos laços e a densidade de uma rede, caracterizando que o conjunto de laços fortes forma uma rede densa e o conjunto de laços fracos forma uma rede menos densa. De acordo com a rede social analisada, a rede da COOPISC possui uma densidade baixa, com um percentual de $30,8 \%$ das ligações possíveis, indicando que os associados não possuem muitos laços com a maioria do núcleo.

A densidade de uma rede também pode ser fator de identificação do potencial de comunicação dentro da rede. De acordo com Silva (2010, p. 58) “[...] a densidade é um índice do potencial de comunicação entre as partes da rede e assim é um índice da quantidade e dos tipos de informação que podem ser trocados teoricamente". O baixo indicador de densidade da rede da COOPISC, relacionado ao fator comunicação entre os agentes, pode ser relacionado ao fato de que alguns piscicultores possuem maior quantidade de laços que os demais, conforme pode ser visualizado na Figura 1. Esses agentes são de extrema importância para a transmissão do conhecimento na rede, mas se retirá-los do fluxo de informações, a aprendizagem na rede pode ser prejudicada pela quebra dos laços, demonstrando que a densidade baixa de relações sobrecarrega alguns indivíduos da rede.

A variável reciprocidade indica o grau de coesão de uma rede, na qual todas as escolhas feitas par a par são mútuas, ou seja, se existe uma transferência recíproca de aprendizagem entre dois membros. Na rede da COOPISC o índice de reciprocidade é de $30,2 \%$, ou seja, as relações são pouco reciprocas e, portanto, tendem a ser menos efetivas. A variável coeficiente de agrupamento (clustering coefficient), constituída pela probabilidade média de que dois nós que possuem um "amigo" em comum estejam conectados diretamente, ou seja, em uma relação de amizade seria representado pelo amigo do amigo, é representado por um percentual de 55\% na rede analisada. Esse percentual de agrupamento é justificado principalmente por se tratar de uma rede com poucos integrantes, em que existe uma relação de troca de experiências entre os produtores.

A variável mais utilizada nas análises de redes sociais é o índice de centralidade de uma rede ou dos agentes que nela estão inseridos. A centralidade permite identificar os atores mais centrais, em termos de estrutura geral da rede. A centralidade de um ator é igual à soma das suas conexões com outros atores. Ao considerar a cadeia como um todo, identificamos que o grau de centralidade de proximidade é de 0,027 , indicando que a maior parte das relações possui uma distância significativa, podendo ser causador de demoras na transferência de informações. A centralidade de intermediação da rede inteira é de 0,138 , sendo que esse valor foi alavancado pelo alto grau de intermediação de seis agentes específicos, conforme análise dos agentes, a seguir.

Na centralidade de intermediação (betweenness), se mede o grau em que o individuo focado se situa como elo entre outros indivíduos que não estão diretamente conectadas (FREEMAN, 1978). A centralidade de intermediação de um nó é calculada através da percentagem de relações que passam por um indivíduo. Os indivíduos ou organizações potencialmente influentes estão posicionados em conexões intermediárias entre os grupos e podem utilizar a influência de um grupo sobre outro, ou servir como uma ligação entre os grupos.

Após a análise geral da rede e da estrutura da rede, apresentada na Figura 1, é interessante verificar os dados de centralidade geral da rede, apresentados na Tabela 4, onde se identificam os agentes com maior poder de centralidade. A centralidade de grau de um agente (total degree centrality) consiste na soma

Persp. online: hum. \& sociais aplicada., Campos dos Goytacazes, 20 (7), 28-42, 2017

seer.perspectivasonline.com.br 
normalizada dos graus de sua linha e coluna. Indivíduos ou organizações que se destacam nesse índice são aqueles que estão ligados a muitos outros e, assim, ter acesso às ideias, pensamentos, crenças de muitos outros. Esses indivíduos são identificados por grau de centralidade relevante na rede social, visto que possuem mais conexões na mesma rede. Segundo Hanneman (2001), se um ator recebe muitas ligações direcionadas a ele, diz-se que ele tem prestígio na rede, ou seja, muitos outros atores buscam compartilhar informações com ele e isso pode indicar sua importância. Os atores que procuram outros, ou seja, os que têm alto grau de saída de ligações, normalmente são atores influentes.

Tabela 4: Grau de Centralidade Total

\begin{tabular}{llc}
\hline $\mathbf{N}^{\mathbf{0}}$ & Cooperado & Valor \\
\hline 1 & Vagner & 48.718 \\
2 & Pedro & 47.436 \\
3 & Marcos & 33.333 \\
4 & Benjamim & 32.051 \\
5 & João & 31.410 \\
6 & Lucas & 16.026 \\
7 & Bernardo & 16.026 \\
\hline
\end{tabular}

Através dos dados da Tabela 4, é possível verificar que a COOPISC possui um grupo de produtores com relações mais homogêneas que compõem o grupo central na transmissão e geração do conhecimento, representado pelos produtores Marcos, Pedro, Benjamim, João, Vagner, Lucas e Bernardo. Estes são os indivíduos centrais na rede, demonstrando ainda que possuem uma concentração de poder alta, que pode ter sido causada pela maior participação desses agentes nas tomadas de decisões e nas atividades da cooperativa, conforme foi visualizado no acompanhamento dos produtores durante a obtenção das respostas aos questionários.

Ao considerar que dentro de uma rede pode haver subgrupos mais próximos e com maior relação de confiança entre os membros, caracterizando, portanto, que pode-se tratar de uma comunidade de prática no interior da rede de aprendizagem, optou-se por analisar o sub-grupo de sete produtores e suas relações pelas variáveis de análise de redes sociais. A Figura 2, a seguir, demonstra a rede de relacionamento de aprendizagem do subgrupo de piscicultores. 


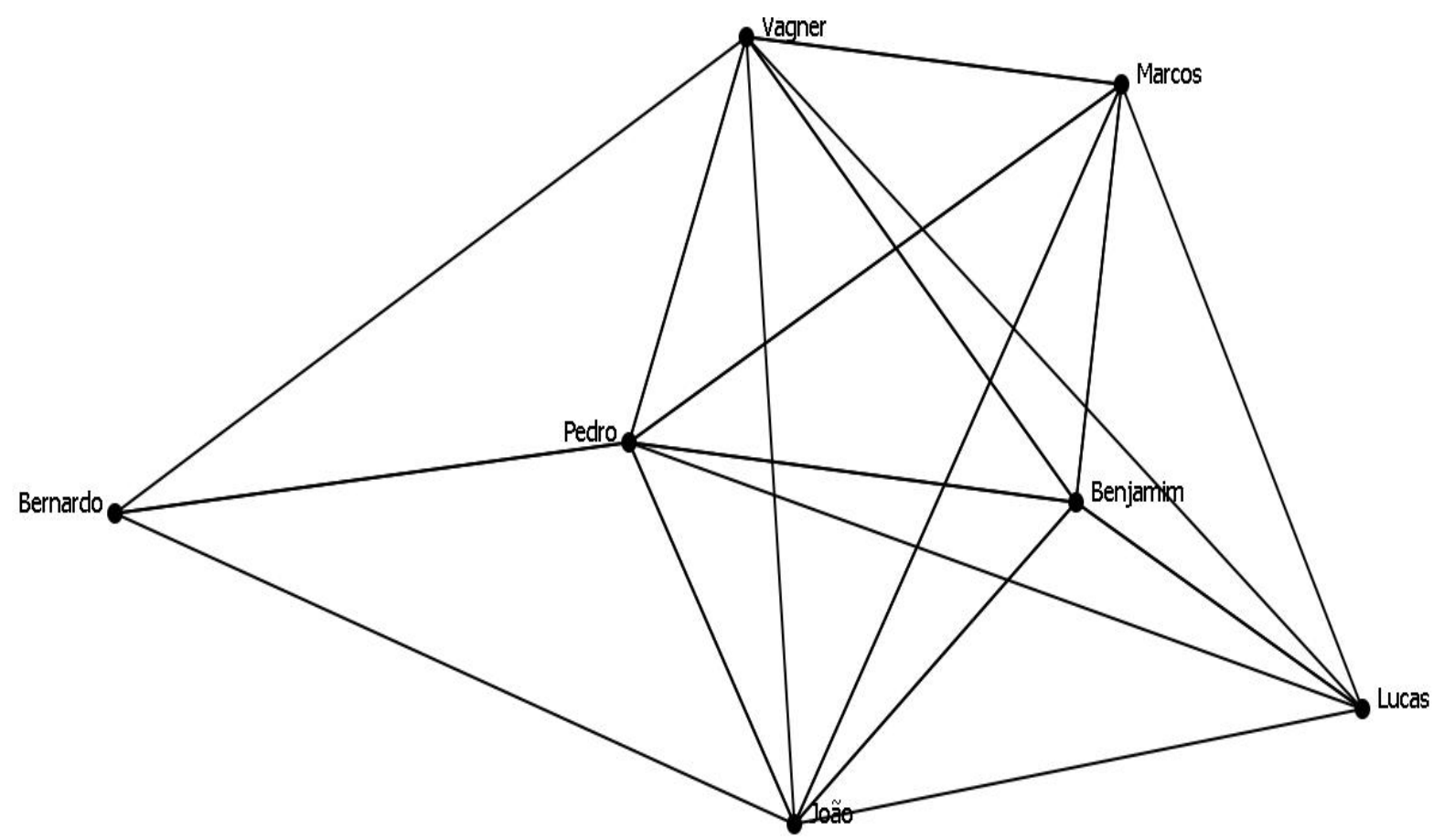

Figura 2: Subgrupo da Rede de relações de aprendizagem da COOPISC

Ao verificar a Figura 2, percebe-se que os integrantes do subgrupo possuem relações mais próximas e coesas com praticamente todos os outros membros. Essa afirmação pode ser confirmada com os dados constantes na Tabela 5.

Tabela 5: Medidas de análise do subgrupo de redes COOPISC

\begin{tabular}{lc}
\hline Medida & Valor \\
\hline Contagem de indivíduos & 7 \\
Numero de ligações & 29 \\
Densidade & 0.690 \\
Reciprocidade & 0.611 \\
Coeficiente de agrupamento & 0.752 \\
Centralidade de Intermediação & 0.103 \\
Centralidade de proximidade & 0.206 \\
\hline
\end{tabular}

Conforme mencionado anteriormente, o subgrupo em análise é composto por sete indivíduos que geram 29 relações de troca de aprendizagem, gerando uma média de 4,1 ligações por membro, uma média alta em relação às análises de rede social. Essa média alta de relações entre os membros é refletida na densidade da rede, com um percentual de $69 \%$, ou seja, do número de ligações possíveis entre os membros, $69 \%$ é efetivada. Ao comparar a densidade da rede de aprendizagem da COOPISC em sua totalidade com o percentual da densidade do subgrupo é possível identificar que na totalidade da rede as relações são mais dispersas e menos intensas, e que esse subgrupo, pela alta intensidade de suas relações internas é mais propenso à troca de experiências e compartilhamento de conhecimentos, visto que cada um dos membros do subgrupo indicou os demais membros como fonte de aprendizagem através de troca de informações e experiências.

O índice de reciprocidade é de aproximadamente $61,1 \%$ das relações, ou seja, predominam no subgrupo as relações mútuas em que existe a troca de conhecimentos e o aprendizado por ambos os membros, garantindo assim a aquisição coletiva de conhecimento entre um conjunto de organizações, conforme proposto por Larsson et al. (1998). As relações de troca de aprendizagem interorganizacional 
obterão maior sucesso no subgrupo do que na rede da COOPISC por completo, que possui reciprocidade de $30 \%$ das relações, visto que a alta reciprocidade do subgrupo demonstra a capacidade de ambos os membros de uma relação aprender, e não somente um indivíduo, demonstrando a importância da reciprocidade em combate ao comportamento oportunista.

Os índices de densidade e de reciprocidade refletem o alto valor do coeficiente de agrupamento (75,2\%). O alto coeficiente de agrupamento é causado pela intensidade e quantidade de relações e também pelo número de integrantes do grupo, sendo que grupos menores tendem a possuir um coeficiente de agrupamento menor e são, portanto, mais propensos a se tornarem fontes de aprendizagem interorganizacional, visto que a proximidade dos membros associa-se a trocas de experiências e confiança nos membros.

Ao considerar os índices de centralidade do subgrupo, esses se apresentaram um pouco maiores comparados ao da rede inteira. O grau de centralidade de proximidade é de 0,284 , indicando que a maior parte das relações possui uma distância significativa, porém ainda menores que ao considerar a rede inteira, que possui o índice de 0,027 . A consequência desse índice maior para o subgrupo é que no interior desta as informações são transferidas com maior agilidade. A centralidade de intermediação do subgrupo é de 0,165 , enquanto da rede inteira é de 0,138 , sendo que esse indicador não possui grandes alterações pelo fato de que os atores com maiores graus de intermediação na rede inteira são exatamente os produtores considerados na análise do subgrupo.

A formação do grupo e o nível de coesão das relações podem ser explicados pelo fato de que todos os 7 envolvidos no subgrupo são integrantes do grupo de fundadores da COOPISC, e pertencem ao percentual de piscicultores que moram na região há mais de 20 anos, gerando condições para que exista um conhecimento da reputação, atitudes e experiências passadas do piscicultor pelos demais membros. Além disso, principalmente por fazerem parte da fundação da cooperativa, estes produtores afirmaram que antes da criação se reuniam frequentemente para discutir assuntos relacionados à atividade da piscicultura, as estratégias de atuação e para troca de experiências de suas propriedades, indicando a presença de uma comunidade de prática reunindo esses 7 produtores.

A análise da rede de relações sociais da COOPISC identificou que existe um subgrupo mais coeso, evidenciado durante a coleta dos dados, e com características de uma CoP. Os resultados mostram que a metodologia de análise de redes sociais pode ser utilizada como uma forma adicional de comprovação de existência de uma CoP. Além desse aspecto, ela é capaz de identificar os indivíduos centrais nas relações da CoP, possibilitando a identificação de fatores importantes no aumento da confiança entre os agentes e na manutenção da cooperação no aprendizado.

Com base na análise realizada, verifica-se que as características da CoP pode ajudar na consolidação da cooperativa, e baseado neste caso, pode-se observar que as reuniões, as trocas de experiências, a transmissão e difusão de conhecimentos através da prática entre o grupo inicial (comunidade) sobre um tema comum (a piscicultura), fizeram com que surgisse a ideia da cooperativa. Após a criação da COOPISC, esses fatores se mantiveram presentes, aumentando o nível de amizade, confiança e cooperação entre os produtores, assim como a confiança na cooperativa. Além de trazer um alto grau de compartilhamento de informações técnicas e de mercado entre os produtores e também pela cooperativa.

\section{CONSIDERÇÕES FINAIS}

O presente estudo relacionou os conceitos de Comunidade de Prática (CoP) e de análise de redes sociais com o objetivo de identificar, caracterizar e analisar a rede social das CoPs na rede formada pelos membros de uma cooperativa brasileira. Para tanto, foi realizado um estudo descritivo com abordagem quantitativa sob a ótica da análise de redes sociais informais de aprendizagem na cooperativa brasileira COOPISC.

A COOPISC, como um todo, não possui características suficientes para classificá-la como uma comunidade de prática, visto que a rede social da cooperativa possui baixa coesão, não aproveita as 
possibilidades existentes de aumentar o compartilhamento de conhecimento e, assim, uma aprendizagem coletiva mais efetiva.

Verificou-se através da metodologia de análise de redes sociais que, no interior da rede social formada pelos produtores participantes, um grupo possuía destaque com maior coesão e maior centralidade. A análise dos indicadores de estrutura demonstrou que esse subgrupo possui alto grau de coesão nas relações, indicando tratar-se de uma propensa CoP. O grupo estudado apresentou as características da CoP tanto antes da formação da cooperativa como também após sua formação. Essas características foram e são fundamentais para essa formação, consolidação e continuidade da cooperativa.

Os benefícios trazidos pela $\mathrm{CoP}$, tais como, o aumento da confiança entre os cooperados, a criação de conhecimento através da troca de experiências, o compartilhamento de informações técnicas e de mercado entre os cooperados, fortalecem os laços entre os produtores, além de fomentar formas de enfrentar as dificuldades advindas da, ainda pouco estruturada, cadeia produtiva de piscicultura no estado do Mato Grosso do Sul. Nesse contexto, a metodologia de análise de redes sociais fornece informações importantes para a identificação dos atores chaves na CoP, aqueles com maior centralidade e maior poder de transferência de conhecimento, podendo ser considerado como uma porta de informações externas.

Apesar dos importantes e exclusivos resultados levantados nesse estudo, houveram alguns fatores que limitaram o aprofundamento das informações, dentre eles, estão a impossibilidade de realizar um grupo focal com os produtores, utilizando perguntas abertas, no intuito de captar informações que através dos questionários não são possíveis, ou que explicariam os resultados obtidos na análise das relações sociais da COOPISC.

No entanto, o objetivo deste estudo não foi o de esgotar o assunto tratado, visto que o mesmo é amplo e pode ser abordado sob diversas óticas. A intenção é colocar o tema CoP no contexto do Agronegócio e demonstrar a importância das redes sociais de cooperação, buscando contribuir e enriquecer as discussões sobre as CoPs e a sua importância para as organizações.

\section{REFERÊNCIAS}

BINOTTO, E. Criação de Conhecimento em Propriedades Rurais no Rio Grande do Sul, Brasil e em Queensland, Austrália. Porto Alegre, RS. Tese de Doutorado. Centro de Estudos e Pesquisas em Agronegócios, UFRGS, 2005, 268 p.

BINOTTO, E. et al. A Comunidade de Prática como ferramenta de criação de conhecimento no contexto do agronegócio. Desenvolvimento em Questão. Ijuí, Brasil: Universidade Regional do Noroeste do Estado do Rio Grande do Sul. v. 5, n. 10, p. 11-42, 2007.

BOYETT, J. H.; BOYETT, J. O guia dos gurus: os melhores conceitos e práticas de negócios. Rio de Janeiro: Campus. 1999, 378 p.

DAVIS, J. H.; GOLDBERG, R. A. A concept of agribusiness. Boston: Division of Research, Graduate School of Business Administration - Harvard University. . 1957, 136 p.

DYER, J.; NOBEOKA, K. 2000. Creating and managing a highperformance knowledge-sharing network: the Toyota case. Strategic Management Journal, v. 21, n. 3, p. 345-367.

FENSTERSEIFER, J. E. Internacionalização e cooperação: dois imperativos para a empresa do terceiro milênio. REAd - Revista Eletrônica de Administração. v. 6, n. 3, p. 1-9, 2000.

FONTES, B. A. S. M.; EICHER, K. A formação do capital social em uma comunidade de baixa renda. REDES- Revista hispana para el análisis de redes sociales. v. 7, n. 2, p. 1-33, 2004.

FREEMAN, L. C. Centrality in Social Networks: Conceptual Clarification. Social Networks. v. 1, n. 3, p. 215-239, 1978. 
GIANEZINI, M. O cooperativismo e seu papel no processo de desenvolvimento local: a experiência das cooperativas agrícolas no médio norte de Mato Grosso. ESAC Economia Solidária e Ação Cooperativa. v. 5, n. $1,2010$.

GRANOVETTER, M. S. The Strength of Weal Ties. American Journal of Sociology, v. 78, n. 6, p. 13601380, 1973.

GROPP, B. M. C.; TAVARES, M. D. G. P. Comunidade de Prática - Gestão de conhecimento nas empresas. São Paulo: Trevisan Editora Universitária. 2006, 120 p.

HANNEMAN, R. A. Introduction to Social Network Methods. Riverside: University of California. 2001.

LARSSON, R. et al. The Interorganizational Learning Dilemma: Collective Knowledge Development in Strategic Alliances. Organization Science, v. 9, n. 3, p. 285-305, 1998.

LEVINSON, N., ASAHI, M. Cross-National Alliances and Interorganizational Learning. Organizational Dynamics, v. 24, n. 2, p. 50-64, 1995.

MARTELETO, R. M. Análise de redes sociais: Aplicação nos estudos de transferência de informação. Ciência da Informação, v. 30, n. 1, 2001, p. 71-81, 2001.

NONAKA, I. TAKEUCHI, H. Criação de conhecimento na empresa. 2. ed. Rio de Janeiro: Campus. 1997.

PARKHE, A. Building trust in international alliances. Journal of World Business, v. 33, n. 4, p. 417-437, 1998.

SCHNEIDER, J. O. Globalização, Desenvolvimento Local Sustentável e Cooperativismo. In: III Encuentro de Investigadores Latinoamericanos de Cooperativismo. São Leopoldo, 1994. Anais...São Leopoldo, Unisinos.

SILVA, A. F. D. Análise de redes sociais informais e o compartilhamento do conhecimento organizacional. Santa Maria, RS. Dissertação de Mestrado. Centro de Ciências Sociais e Humanas, Universidade Federal de Santa Maria, 2010, 103 p.

SOUZA-SILVA, J. C.; SCHOMMER, P. C. A pesquisa em comunidades de prática: panorama atual e perspectivas futuras. Organizações e Sociedade, v. 15, n. 44, p. 105-127, 2008.

TSANG, E. Organizational Learning and the Learning organization: A dichotomy between descriptive and prescriptive research. Human Relations, v. 50, n. 1, p. 73-89, 1997.

UZZI, B. Social structure and competition in interfirm networks: The paradox of embeddedness. Administrative Science Quarterly. v. 42, n. 1, p. 35-67, 1997.

WENGER, E.; MCDERMOTT, R.; SNYDER, W. M. Cultivating Communities of Practice: a guide to managing knowledge. Boston: Harvard Business School Press. 2002, 316 p.

Persp. online: hum. \& sociais aplicada., Campos dos Goytacazes, 20 (7), 28-42, 2017

seer.perspectivasonline.com.br 